\title{
Evaluation of CRAFT as a Tool for Caries Risk Assessment in 3- to 6-year-old Children and its Validation against Alban's Test: A Pilot Study
}

\author{
Jagruti H Thakur ${ }^{1}$, Subhadra $\mathrm{HN}^{2}$, Ashwin Jawdekar ${ }^{3}$
}

\begin{abstract}
Introduction: Dental caries is a multifactorial disease and a dynamic process that can be prevented and reversed during the early stage. Risk assessment is an important step in decision-making and treatment planning. There are no valid tools available for the Indian population. Caries risk assessment for treatment (CRAFT) is a chairside tool for caries risk assessment and management. Valid, reliable, economical, and chairside caries risk assessment tool is the need of the hour for general and pediatric dentists.

Aim: To evaluate CRAFT as a tool for caries risk prediction among 3-years to 6-years-old children and to validate it against Alban test.

Materials and methods: A pilot study was conducted, including forty 3-years to 6-years-old children. Salivary samples were collected and inoculated on B.C.G.-Dextrose Agar. Caries activity was assessed using Alban test. Their parents/guardians completed the CRAFT assessment in entirety.

Results: The data were tabulated and subjected to suitable statistical analysis. High positive correlation between CRAFT scores and Alban's test (Spearman's Rho $=0.874$ ) was found.

Conclusion: CRAFT scores were highly correlated with Alban scores in 3- to 6-year-old children. CRAFT could be successfully employed as a reliable, economical, chairside, and clinically feasible risk assessment tool with further research on a larger sample size.

Clinical significance: CRAFT can provide a framework for the clinician for preventive care and enhance patient-participation for CRA.

Keywords: Alban's test, Caries risk assessment, Caries risk assessment for treatment, Early childhood caries.

International Journal of Clinical Pediatric Dentistry (2019): 10.5005/jp-journals-10005-1698
\end{abstract}

\section{INTRODUCTION}

Early childhood caries (ECC) poses to be a major problem worldwide. ${ }^{1}$ Dental caries is an initially reversible and chronic disease process with a known multifactorial etiology. ${ }^{2}$ Primary prevention or treatment of early lesions is important for halting the progression of the lesions to irreversible caries. ${ }^{3}$ This can be achieved by assessing the true caries risk of an individual. Various salivary tests have been used to detect caries activity based on the bacterial count or acidogenic potential. ${ }^{4}$ Such tests establish the current caries activity but do not predict future risk.

"Risk is a probability that an event will occur." ${ }^{5}$ Caries risk assessment (CRA) is "prediction of future caries based on the diagnosis of current disease by evaluation of risk and protective factors for making evidence-based clinical decisions." CRA should be included in a treatment plan in order to assist the clinician in the decision making process concerning treatment, recall appointments, and need for additional diagnostic procedures. ${ }^{6}$ There are many CRA tools available for the developed nations, but the same is not validated for the Indian population.

A valid, reliable, economical, and chairside CRA tool for the Indian population, is needed.

\section{CRAFT-Conceptual Framework}

"Caries risk assessment for treatment (CRAFT)" is a proposed simple, chairside, noninvasive, four-point approach proposed for the management of caries based on risk assessment. For ascertaining the caries-risk in children, parents can be interviewed using the CRAFT questionnaire. CRAFT is available as a physical and a digital tool.
${ }^{1-3}$ Department of Pediatric and Preventive Dentistry, Dr GD Pol Foundation's YMT Dental College and Hospital, Navi Mumbai, Maharashtra, India

Corresponding Author: Jagruti H Thakur, Department of Paediatric and Preventive Dentistry, Dr GD Pol Foundation's YMT Dental College and Hospital, Navi Mumbai, Maharashtra, India, Phone: +91 9004089897, e-mail: drjagrutithakur@gmail.com

How to cite this article: Thakur JH, Subhadra HN, Jawdekar A. Evaluation of CRAFT as a Tool for Caries Risk Assessment in 3- to 6-yearold Children and its Validation against Alban's Test: A Pilot Study. Int J Clin Pediatr Dent 2019;12(6):538-542.

Source of support: Nil

Conflict of interest: None

It includes four parameters: diet, decay, fluoride exposure, and other factors. Based on the available information, caries-risk can be determined over a range (very low/no, low, moderate, high). A green star is used to indicate "safety" while a red star for the "risk" (Fig. 1).

Based on the risk as ascertained from the CRAFT, ageappropriate home measures can be recommended from below.

- Reduce sugar intake to less than two exposures per day and preferably at meals.

- Stop bottle-feeding.

- Consider non-syrupy medicines (recommendation to consult the doctor for the same). 


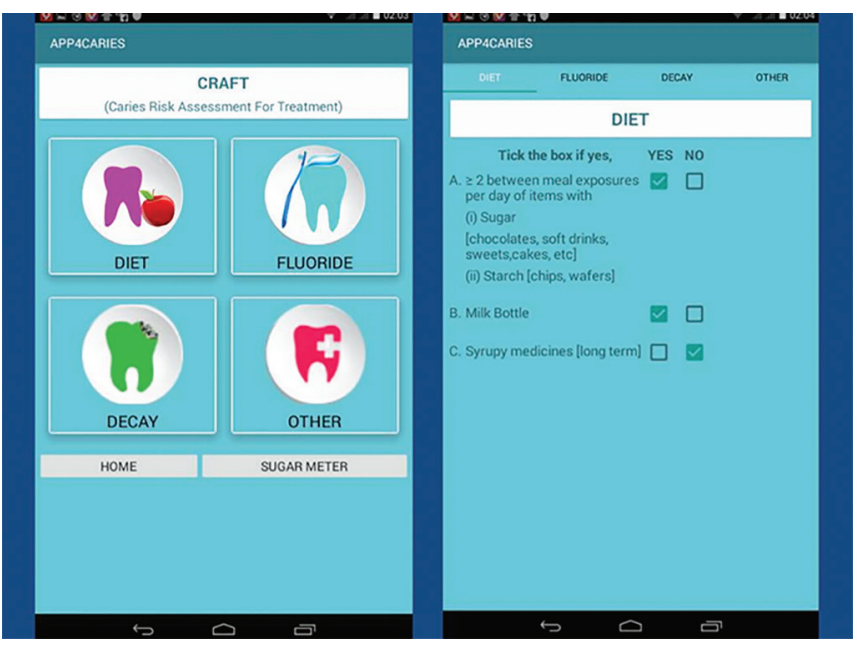

Fig. 1: CRAFT (screenshot of the digital tool)

- Use appropriate toothpaste (non-fluoride for less than 2-yearsolds, junior fluoride for 2- to 6-year-olds, and regular fluoride for all above 6 years), and fluoride mouth rinse (for those above 6 years).

- Take treatment for untreated carious teeth of the child.

- Take treatment for untreated carious teeth of the parent/s.

- Consult your family physician/pediatrician/specialist regarding recurrent illnesses, allergies, etc.

- Strengthen the teeth enamel with the use of remineralizing agents (consult your dentist for the same).

- Use hydrating agents or sugar-free chewing gums (consult your dentist for the same).

- Have teeth alignment corrected (consult your dentist for the same).

Additionally, a treatment plan with the use of minimum interventional and restorative approaches can be integrated with CRAFT, as shown below:

Snyder test with Alban's modification ${ }^{7}$ has been used extensively for the quantitative evaluation of acidogenic potential of the saliva. ${ }^{8}$ It is an easily available and cost-effective method, which can be used in place of quantitative estimation of streptococcus mutans.

In children with different caries levels, caries activities may differ. Risk considerations are based on wider etiological factors and not just the present status of the activity. However, the (present) activity and (future) risk can be correlated to get a better picture in terms of susceptibility. Furthermore, an attempt to associate the two can help validate a newly developed tool, such as the one presented here, CRAFT.

Research on CRA tools in the Indian context is scarcely reported. The present investigation was carried out to assess the implementation of the new CRA tool CRAFT and its validation against the Alban's test in 3- to 6-year-old children.

\section{Materials and Methods}

Caries risk assessment for treatment (CRAFT) being a new CRA tool, a pilot study was planned. Ethical clearance was obtained from the institutional review board. Consent was obtained from the parents of children participating in the study. Fifty healthy children of age group 3 to 6 years were randomly chosen from those visiting the Department of Pediatric and Preventive Dentistry. Untoward contamination of the medium during the study led to

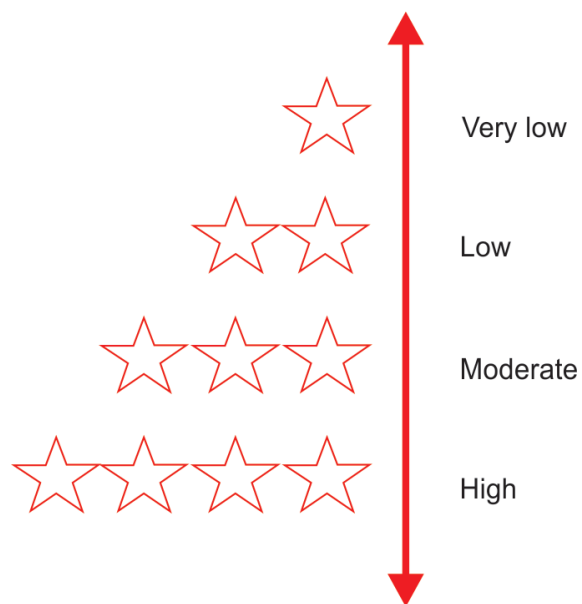

Fig. 2: CRAFT scores

the elimination of eight children leading to a final sample size of forty-two.

Healthy children age 2 to 6 years were chosen for the study. Children with a recent history of, or under antibiotic treatment were excluded from the study. The parents/guardians of all the fifty children were asked to complete the CRAFT assessment in entirety. The digital tool APP4CARIES was used in this study. Risk was assessed using CRAFT as very low, low, moderate, high (Fig. 2, Tables 1 and 2).

The Alban's test is a simplified substitute for the colorimetric Snyder test, which uses the lesser medium as compared to the Snyder's test. Alban's test was used to assess caries activity at various time points of observation. The preparation of the Snyder medium was done using commercially available-BCG-Dextrose Agar (Snyder Test Agar: HiMedia Laboratories Pvt Ltd, Mumbai). $65 \mathrm{~g}$ of the Snyder test agar was suspended in $1 \mathrm{~L}$ of distilled water. The mixture was boiled, and each Snyder test tube was dispensed with $5 \mathrm{~mL}$ of media and autoclaved at $121^{\circ} \mathrm{C}$ at $15 \mathrm{lbs}$, for 15 minutes. The contents of the tube were allowed to solidify

Table 1: CRAFT categories

\begin{tabular}{lc} 
Caries risk assessment for treatment (CRAFT) \\
\hline Diet $\quad$ Decay status
\end{tabular}

- $\geq 2$ exposures per day of sugar or starch containing food items between meals (Yes/No)

- Bottle feeding (Yes/No)

- Long-term exposure to syrupy medicines (Yes/No)

Fluoride exposure

- Use of fluoridated toothpaste (Yes/No)

- Use of fluoride mouthrinse (Yes/No)

- Professional six monthly fluo- - Malocclusion/crowding (Yes/ ride application (Yes/No)

- Living in area with high/ optimally fluoridated water (Yes/No)
- Present untreated caries (Yes/ No)

- Past treated caries (Yes/No)

- Parent's caries status (Yes/No)

Other factors

- Conditions related to suppressed immunity (asthma, allergies, recurrent infections, etc.) (Yes/No)

- Hypomineralization (Yes/No) No)

- Hyposalivation (Yes/No) 
before storage in the refrigerator. Prior to saliva collection, Snyder tubes were rolled between hands to bring the temperature close to body temperature. Following this, the open end of the tube was momentarily exposed to flame to sterilize the tube opening and prevent any untoward sample contamination. Unstimulated, midmorning salivary samples were collected just sufficient to cover the superior surface of the medium. Evaluation of caries activity employing Alban's test was done on the basis of length of color change following incubation at $35^{\circ} \mathrm{C}$ to $37^{\circ} \mathrm{C}\left(95-99^{\circ} \mathrm{F}\right)$ every 24,48 , 72, and 96 hours (Table 3 and Fig. 3). Caries activity was assessed based on cumulative color change at the end of 96 hours. The caries activity was divided into 4 categories based on the color changes obtained in the Snyder tubes after 96 hours.

\section{Variables}

Both CRAFT score and Alban's score were recorded as categorical variables, but for correlation purpose, the same was converted to quantitative (continuous) variable.

Table 2: Example of CRAFT-based office recommendations

\begin{tabular}{|c|c|}
\hline Preventive measures in the office & $\begin{array}{l}\text { Minimum interventional and } \\
\text { restorative measures }\end{array}$ \\
\hline Fluoride application & $\begin{array}{l}\text { Surface modification (making } \\
\text { non-accessible surfaces accessible } \\
\text { for easy plaque-elimination) }\end{array}$ \\
\hline Pit and fissure sealants & $\begin{array}{l}\text { Reduction and stabilization } \\
\text { of caries activity with interim } \\
\text { restorations }\end{array}$ \\
\hline Oral prophylaxis & $\begin{array}{l}\text { Sealing caries (sealants, preventive } \\
\text { resin restorations, preformed } \\
\text { metal crowns) }\end{array}$ \\
\hline $\begin{array}{l}\text { Dietary and hygiene advice and } \\
\text { recalls }\end{array}$ & $\begin{array}{l}\text { Partial/stepwise excavation and } \\
\text { restorations (glass-ionomer and } \\
\text { composite resins) }\end{array}$ \\
\hline
\end{tabular}

Table 3: Alban's scoring criteria

\begin{tabular}{ll}
\hline Color change & Score \\
\hline No color change (negative) & - \\
Beginning color change (from top medium down) & + \\
One-half color change (from top down) & ++ \\
Three-fourths color change (from top down) & +++ \\
Three-fourths color change (from top down) & ++++ \\
\hline
\end{tabular}

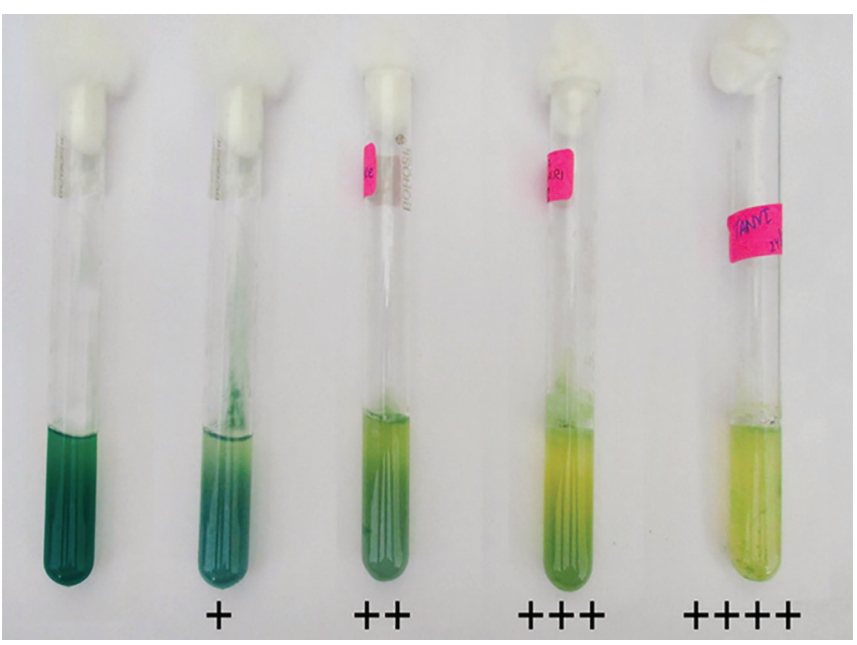

Fig. 3: Alban's scores based on color change

\section{Statistical Analysis}

Spearman's correlation test was used to establish the association between cumulative Alban's score at the end of 4 days and CRAFT scores.

\section{Results}

Of the 42 children, 19 were girls and 23 boys. The mean age of children was $4.91 \pm 0.91$ years. About $33.33 \%$ (14) had High CRAFT scores, $50 \%$ (21) of the participants had a Moderate CRAFT score, and $16.7 \%$ (7) had Low CRAFT scores, and $0 \%$ fell in the very low category (Table 4).

The mean cumulative score for Albans was $8.36 \pm 3.93$, with the lowest being zero to the highest of 17 (Table 5).

The cumulative Alban's score increased with an increase in the CRAFT score.

A scatter plot showing the linear relationship between CRAFT score and Alban's score (Spearman's Rho $=0.874 ; p<0.001$ ) showed a high positive correlation between the two (Fig. 4).

\section{Discussion}

The study was conducted in the Department of Pediatric and Preventive Dentistry in Navi Mumbai. Children 3 to 6 years of age were recruited for the following reasons.

Children in this age group are easily accessible. By this age, all the primary teeth would have erupted, and more or less the dietary habits would have established. Risk-based caries management is

Table 4: CRAFT score distribution

\begin{tabular}{lcc}
\hline CRAFT score & Number of children & Percent \\
\hline Very low & 0 & 0 \\
Low & 7 & 16.7 \\
Moderate & 21 & 50.0 \\
High & 14 & 33.3 \\
Total & 42 & 100.0 \\
\hline
\end{tabular}

Table 5: Cumulative Alban's score

\begin{tabular}{llllll}
\hline & $N$ & Minimum & Maximum & Mean & Std. deviation \\
\hline $\begin{array}{l}\text { Cumulative } \\
\text { score }\end{array}$ & 42 & 2 & 17 & 8.36 & 3.931 \\
\hline
\end{tabular}

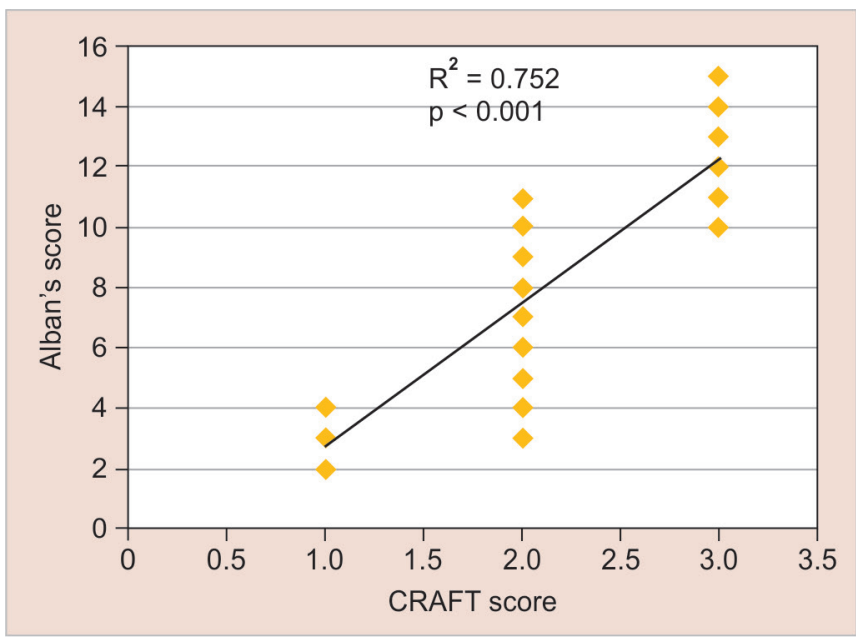

Fig. 4: Correlation between CRAFT score and cumulative Alban's score 
important for this age group to prevent caries in the permanent dentition and would decrease the morbidity associated with caries.

The present study was a pilot investigation conducted that compared CRAFT, a novel tool, with the well-known Alban's test. Alban's test has been widely used in literature to measure the acidogenic potential, which is a proxy measure for the bacterial activity of caries-producing microorganisms like streptococcus mutans and lactobacilli. ${ }^{5}$ We also considered using CRT (Ivoclar) bacteria, but the costs were found to be too high.

The overall CRAFT scores indicate that half of the participants (50\%) fell in the moderate scores category. Also, there was no representation for the very low category. This is possibly due to the presence of the major etiological risk factors causing dental caries viz. diet rich in sugar, burden of untreated decay, low exposure to fluoride, and other factors such as infectious diseases, malnutrition, etc., in the study population. This is in accordance with other studies reported in the literature. ${ }^{9,10}$ In developing countries like India, where the population has more opportunities to consume free sugars and other fermentable carbohydrates the prevalence of decay is increasing. ${ }^{11}$ Very few children receive 6 monthly, fluoride application, moreover, they do not receive centralized fluoridated water supply. ${ }^{12}$ CRAFT included the most important etiological factors as a customized preventive plan based on the four domains are evidence-based.

Existing untreated dental caries gives an estimate of the current bacterial load and caries activity. ${ }^{13}$ In general, the best indicator of future caries risk is the past caries experience. ${ }^{14}$ CRAFT includes both these components; also it includes vertical transmission of caries. The major reservoirs from which the infants acquire MS is their mother. ${ }^{15} \mathrm{~A}$ study by Berkowitz concluded that the frequency of infant infection reduced 9 times when the colony-forming units reduced from $10^{5}$ to $10^{3} .{ }^{16}$ Presence of other risk factors such as crowding, low immunity, or hyposalivation further increases the caries risk. ${ }^{17-19}$

The mean Alban's score was 8.26 (2-15). Frequent snacking in between meals, existing untreated caries, hyposalivation, reduced immunity, and absence of regular use of topical fluorides may have resulted in high mean Alban's score. Caries risk and caries activity are both high in the presence of these etiological factors.

We got a statistically significant very high correlation, which confirms that children with high caries activity present with higher caries risk.

Various CRA tools such as CAMBRA, CAT, and Cariogram are amongst the widely used tools in the developed world. ${ }^{20}$ Most of these tools have been tested for the developed nations, and their utilization in the Indian context has not been studied adequately.

Although Western literature reports socioeconomic factors, migrating population, ethnic backgrounds as important determinants for dental caries, the same for the Indian population have not yet been established. ${ }^{21,22}$ The dietary habits, oral hygiene practices, dental care differ significantly in the Indian context. Furthermore, this tool is developed for the caries risk assessment of an individual and not as an epidemiological tool.

Various CRA tools have microbial activity test as an integral part of the system. ${ }^{23}$ However, these tests not only increase the costs but also makes it more cumbersome and time-consuming for the dentist; hence, they were excluded from CRAFT. A very high correlation between CRAFT and Alban score emphasizes that although microbial testing is essential in most clinical situations if excluded, could still give sufficient idea about the caries risk.

\section{LIMITATIONS}

This being a pilot study was based on a convenient sample, and therefore, there was no representation from the very low category of CRAFT scores. The categorical variables were quantified for statistical purposes. Moreover, the generalizability of a tool cannot be ascertained with a single investigation. Therefore, multicentric studies on large sample with different age groups are necessary to corroborate the claims made by us.

\section{Conclusion}

In the present study, CRAFT and Alban test scores of 3- to 6-yearold children showed a very high correlation. CRAFT has potential as a caries risk assessment tool for the Indian population. Further prospective studies are needed to substantiate the claim.

\section{Clinical Significance}

Caries risk assessment is widely recommended for patient-tailored, prevention-focused caries management. CRAFT can provide a framework for the clinician for preventive care and enhance patientparticipation in the same.

\section{Manufacturer Name}

Snyder test agar: HiMedia Laboratories Pvt Ltd, Mumbai.

\section{ACKNOWLEDGMENTS}

Authors would like to extend their thanks to staff of the Department of Oral Pathology and Microbiology for their help.

\section{References}

1. Kassebaum NJ, Smith AG, Bernabé E, et al. GBD 2015 Oral Health Collaborators. Global, regional, and national prevalence, incidence, and disability-adjusted life years for oral conditions for 195 countries, 1990-2015: a systematic analysis for the global burden of diseases, injuries, and risk factors. J Dental Res 2017;96(4):380-387. DOI: $10.1177 / 0022034517693566$.

2. Pitts NB. Are we ready to move from operative to non-operative/ preventive treatment of dental caries in clinical practice? Caries Res 2004;38(3):294-304. DOI: 10.1159/000077769.

3. Featherstone JD. Dental caries: a dynamic disease process. Aust Dent J 2008;53(3):286-291. DOI: 10.1111/j.1834-7819.2008.00064.x.

4. Guo L, Shi W. Salivary biomarkers for caries risk assessment. J Calif Dent Asso 2013;41(2):107-118.

5. Burt BA. Concepts of risk in dental public health. Community Dent Oral Epidemiol 2005;33(4):240-247. DOI: 10.1111/j.16000528.2005.00231.x.

6. Young DA, Featherstone JD. Implementing caries risk assessment and clinical interventions. Dent Clin North Am 2010;54(3):495-505. DOI: 10.1016/j.cden.2010.04.002.

7. Resta G, Pozzoli GA, Monestiroli P, et al. The Snyder test modified by Arthur Alban. Prev Stomatol 1982;8(2):7-9.

8. Baca P, Parejo E, Bravo M, et al. Discriminant ability for caries risk of modified colorimetric tests. Med Oral Patol Oral Cir Bucal 2011;16(7):e978-e983. DOI: 10.4317/medoral.17358.

9. Kutsch VK, Young DA. New directions in the etiology of dental caries disease. J Calif Dent Assoc 2011;39(10):716-721.

10. Simón-Soro A, Mira A. Solving the etiology of dental caries. Trends Microbiol 2015;23(2):76-82. DOI: 10.1016/j.tim.2014.10.010.

11. Moynihan P, Petersen PE. Diet, nutrition and the prevention of dental diseases. Public Health Nutr 2004;7(1a):201-226. DOI: 10.1079/ PHN2003589. 
12. Bali RK, Mathur VB, Talwar BB, et al. National oral health survey and fluoride mapping 2002. Dental Council of India (DCl); 2004.

13. Powell LV. Caries prediction: a review of the literature. Community Dent Oral Epidemiol 1998;26(6):361-371. DOI: 10.1111/j.16000528.1998.tb01974.x.

14. Li Y, Wang W. Predicting caries in permanent teeth from caries in primary teeth: an eight-year cohort study. J Dent Res 2002;81(8): 561-566. DOI: 10.1177/154405910208100812.

15. da Silva Bastos VD, Freitas-Fernandes LB, da Silva Fidalgo TK, et al. Mother-to-child transmission of Streptococcus mutans: a systematic review and meta-analysis. J Dent 2015;43(2):181-191. DOI: 10.1016/ j.jdent.2014.12.001.

16. Berkowitz RJ. Acquisition and transmission of mutans streptococci. CDA 2003;31(2):135-138.

17. Hafez HS, Shaarawy SM, Al-Sakiti AA, et al. Dental crowding as a caries risk factor: a systematic review. Am J Orthod Dentofacial Orthop 2012;142(4):443-450. DOI: 10.1016/j.ajodo.2012.04.018.
18. Lamont RJ, Hajishengallis GN, Jenkinson HF. Oral Microbiol Immunol. ASM press; 2014

19. Lenander-Lumikari M, Loimaranta V. Saliva and dental caries. Adv Dent Res 2000;14(1):40-47. DOI: 10.1177/08959374000140010601.

20. Tellez M, Gomez J, Pretty I, et al. Evidence on existing caries risk assessment systems: are they predictive of future caries? Community Dent Oral Epidemiol 2013;41(1):67-78. DOI: 10.1111/cdoe.12003.

21. Petersen PE. Sociobehavioural risk factors in dental cariesinternational perspectives. Community Dent Oral Epidemiol 2005 Aug;33(4):274-279. DOI: 10.1111/j.1600-0528.2005.00235.x.

22. Peres MA, Peres KG, De Barros AJ, et al. The relation between family socioeconomic trajectories from childhood to adolescence and dental caries and associated oral behaviours. J Epidemiol Community Health 2007;61(2):141-145. DOI: 10.1136/jech.2005.044818.

23. Bratthall D, Hänsel Petersson G. Cariogram-a multifactorial risk assessment model for a multifactorial disease. Community Dent Oral Epidemiol 2005;33(4):256-264. DOI: 10.1111/j.1600-0528.2005.00233.x. 